\title{
DES FRONTIERES DE LA COMMUNICATION: ENTRE L'ORALISME ET LA LANGUE DES SIGNES
}

\author{
ONOFRE, Eduardo Gomes ${ }^{1}$ \\ FERNÁNDEZ, Sandra Jacqueline Meza² \\ SARGSYAN-SABLONG, Anna ${ }^{3}$
}

\section{RÉSUMÉ}

L'objectif principal du présent article est discutir les frontières de la communication, entre l'oralisme et la langue des signes. Pour cela, nous avons réalisé d'entretiens semi-directifs avec deux jeunes sourds français qui partagent de manière différente leurs relations quotidiennes avec des entendants. Les entretiens ont été réalisés, en septembre 2013, dans un établissement d'enseignement spécialisé pour des sourds à la région du Poitou-Charentes, en France. Les résultats ont indiqué que les avancées des nouvelles technologies de l'information et de la communication, par exemple l'implant cochléaire, amènent les sourds dans une zone frontière. Les sourds qui ont l'implant cochléaire ou appareil auditif, tendent à percevoir les sons car le but principal de ces technologies est de favoriser l'oralisation. Cependant, il faut dire que cette compréhension des sons ne donne pas aux sourds la condition d'entendants.

Mots-clés: Sourds - Frontière - Langue des signes - Oralisme - Communication.

\section{RESUMO}

O objetivo principal do presente artigo é discutir as fronteiras da comunicação, entre 0 oralismo e a língua de sinais. Para tanto, realizamos uma entrevista semi estruturada, em setembro de 2013, com dois jovens surdos franceses que vivem, de maneira diferente, suas relações cotidianas com os ouvintes. As entrevistas foram realizadas, em setembro de 2013, em um estabelecimento de ensino especializado para surdos na região de Poitou-Charentes, França. Os resultados indicaram que os avanços das novas tecnologias de informação e comunicação, a exemplo do implante coclear, levam os surdos a uma zona fronteiriça. Os surdos que utilizam o implante coclear ou aparelhos auditivos tendem a perceber os sons por-

\footnotetext{
${ }^{1}$ Professor da Universidade Estadual da Paraíba (UEPB). Programa de Pós Graduação em Ensino de Ciências e Educação Matemática (PPGECEM).Email: eduonofre@gmail.com

${ }^{2}$ Professora do departamento de Estudos Pedagógicos da Faculdade de Filosofia e Ciências Humanas da Universidade do Chile. Email: smeza@u.uchile.cl

${ }^{3}$ Presidente da Associação Lab-Inclusion - França. Email: anna.sargsyan@yahoo.fr
} 
que o principal objetivo dessas tecnologias é favorecer o oralismo. Entretanto, precisamos dizer que tal compreensão dos sons não dá aos surdos a condição de ouvintes.

Palavras-chave: Fronteira - Língua de sinais - Oralismo - Comunicação.

\section{INTRODUCTION}

Aujourd'hui, les études sur les frontières incluent des questions plus larges, liées au capitalisme international, aux questions économiques, aux questions géographiques et territoriales ainsi que des questions touchant la vie au quotidien, l'éducation et les formes de communication. Il nous faut mettre l'accent sur notre point de départ, nous partons de la présupposition que toutes les frontières ont leur trace symbolique. Comme nous dit Augé (2009, p. 11) "si la notion de frontière est 'bonne à penser', c'est qu'elle est au cœur de l'activité symbolique qui, dès l'apparition du langage, si l'on suit Lévi-Strauss, s'est employée à faire signifier l'univers, à donner un sens au monde pour le rendre visible". Néanmoins, nous percevons qu'il existe des frontières plus symboliques que d'autres. Dans le cas des acteurs sociaux touchés par une surdité, la frontière de la communication et de la langue entre l'oralisme et la langue des signes tend à se redessiner ou à se déplacer. Ainsi, dans cet article, nous discuterons des frontières entre l'être entendant et l'être sourd, ainsi que des frontières de la communication et de la langue qui nous renvoient au droit de l'acteur social sourd de choisir entre l'oralisme et la langue des signes, comme une forme de communication avec les autres et le monde.

Nous travaillons avec deux acteurs sociaux sourds qui ont fait une intervention chirurgicale, l'implant cochléaire. La trace qui différencie ces deux acteurs sociaux, c'est que l'un expérimente encore le passage d'un monde visuel, à la fois silencieux, à un monde qui apprécie les sons, tandis que l'autre a choisi de vivre sa surdité sans plus éprouver le monde des en- tendants. Comme le dit Mottez (2006, p. 52) "alors que pour le spécialiste, il n'y a d'action valable que celle qui porte sur le corps du handicapé, pour le handicapé, il s'agit en effet et à l'inverse, afin de trouver place et se faire accepter, d'agir sur le corps social".

Les sources employées dans cet article sont issues de la littérature sociologique et historique sur les frontières liées aux formes de communication, notamment des personnes sourdes, ainsi que d'entretiens semi-directifs réalisés en septembre 2013 avec les deux jeunes sourds français qui partagent de manière différente leurs relations quotidiennes avec des entendants. Les entretiens ont été réalisés dans une institution spécialisée pour les sourds à la région du PoitouCharentes, en France.

\section{ENTRE L'ORALISME et LA LANGUE DES SIGNES: LES ENJEUX DES DROITS CHEZ LES SOURDS}

Tout d'abord, il nous faut dire que parler de différence provoque toujours une problématisation sur l'opposition entre normalité et anormalité, ainsi qu'une problématisation sur la conception de normalité. La conception de la différence dans une société est en elle-même un processus produisant des conflits quant à l'interprétation de l'autre différent dans le discours de la classe dominante. Par exemple, la configuration d'être entendant est déjà une simple référence à une normalisation en ce qui concerne l'audition. De cette façon, pour plusieurs professionnels de la société actuelle, dans le domaine de l'éducation et de la santé, être sourd signifie ne pas parler, avoir des difficultés 
à s'exprimer, être un "sourd-muet". Ainsi, la société actuelle a développé des techniques sophistiquées qui peuvent développer l'audition des personnes sourdes, par exemple l'implant cochléaire et toutes sortes d'appareillages auditifs. Ces techniques ont comme principal objectif de faciliter aux sourds l'apprentissage de la lecture labiale et de l'oralisme. S'agissant de la surdité, nous mettons l'accent sur deux différentes formes de communication utilisées par les personnes sourdes pour communiquer avec le monde et les autres : I'oralisme et la langue des signes.

En ce qui concerne la méthode de I'oralisme, nous disons qu'elle est trop utilisée par les éducateurs et les professionnels de la santé. Cette méthode est perçue par la société actuelle comme une procédure qui donnera au sourd ce qui lui manque: I'audition. Ainsi, la société occidentale actuelle perçoit la surdité comme une déficience auditive qui se quantifie par la perte de décibels. De cette façon, I'on classifie les sourds en quatre catégories de surdité: légère, moyenne, sévère et profonde. Dans ce sens, la société actuelle définit le "handicap auditif" ou la "déficience auditive", selon Zribi et PoupéeFontaine (2004, p.149) comme:

Déficience auditive légère: perte moyenne comprise entre 20 et 40 décibels; il y a perception de la parole normale mais des difficultés apparaissent si la voix est faible. Déficience auditive moyenne: perte moyenne comprise entre 40 et 70 décibels; il y a perception si la parole est forte; les difficultés sont nettes sans appareillage. Déficience auditive profonde: plus de 90 décibels; si l'enfant n'est pas appareillé et $s^{\prime} i l$ ne reçoit pas une éducation spéciale, il est mutique ; à plus de 100 décibels, la surdité est totale.

En accord avec la conception cidessus, la société actuelle définit les sourds en utilisant toujours comme principale référence la déficience auditive. De cette façon, la société développe des ressources qui vont proposer au sourd l'acquisition de la voix. Cette manière de voir les personnes sourdes conduit à la suprématie de l'oralisme. Cette question nous renvoie à la thématique conflictuelle sur la diversité humaine dans une société qui impose des valeurs liées à la production et à la consommation. Ces relations nous montrent aussi le pouvoir d'une société des entendants sur les personnes sourdes.

D'après nous, la méthode de I'oralisme ne peut être perçue comme la voie qui placera les sourds dans la catégorie des entendants. L'acteur social sourd avec un implant cochléaire ou des appareils auditifs aura toujours besoin de consulter des orthophonistes. Il y a de nombreux éducateurs qui pensent l'oralisme comme la principale ressource pour promouvoir l'inclusion sociale des sourds. La méthode de l'oralité à figé le sourd sur la frontière entre l'être entendant et l'être sourd.

Il nous faut aborder les personnes sourdes avec une nouvelle vision ethnique et les considérer comme un groupe social ayant sa propre culture et sa propre langue, dont se différencie la langue des entendants. Alors, dans notre recherche, nous considérons les personnes sourdes comme des individus qui perçoivent le monde par leurs expériences visuelles et utilisent le langage des signes pour mieux s'intégrer dans la société. Ainsi, l'identité sourde ne se réduit pas à un corps déficient qui doit être moulé sur celui de l'acteur social entendant, comme l'affirme la société dominante. L'identité sourde est toujours reliée au monde visuel, gestuel, qui est loin d'un monde où les perceptions sonores ont une forte influence dans les relations quotidiennes. Comme le dit Le Breton (2011, p. 158) 
On peut vivre sans voix, ne l'avoir jamais entendue, et participer pleinement au lieu social. Les sourds possèdent une culture propre, un style de vie. Le fait d'être confrontés à des personnes qui ignorent leur langue signée et opposent à leur encontre des représentations négatives ajoute aux difficultés de communication, mais ces entraves sont celles soulevées dans toute rencontre interculturelle.

En vérité, la société entendante dominante refuse de reconnaître les traces identitaires d'un groupe minoritaire. Être minoritaire, selon Wirth cité par Juteau (1999, p. 134):

C'est avoir un statut qui comprend deux dimensions, l'une objective, I'autre subjective. La dimension objective comporte deux aspects: la présence de marques physiques ou culturelles distinctives et l'accès inégal aux ressources économiques, politiques et juridiques dans une société. La dimension subjective, qui découle des pratiques discriminatoires, implique un sentiment de persécution et un complexe d'infériorité qui peuvent donner lieu à une prise de conscience de la situation de domination.

Alors, reconnaître, comprendre, accepter que la langue des signes est la première langue des sourds va favoriser la construction de l'identité ainsi que la socialisation de ces individus que la société appelle encore "muets". Comme nous bien dit Watier (2002, p. 77) "la compréhension est un outil méthodologique de la connaissance historique, mais, plus fondamentalement encore, elle est une modalité mise en œuvre par les individus pour interagir dans la société".

Il faut noter par ailleurs qu'entre la frontière de l'oralisme et de la langue des signes, s'installe le conflit qui dans ce cas, à notre avis, est lié au développement des nouvelles technologies d'information et de communication, ainsi que des sciences médicales. Selon Augé (2009, p. 12) "I'histoire politique de la planète semble mettre en cause les frontières traditionnelles à I'heure où un marché libéral mondial se met en place et où les technologies de la communication semblent chaque jour effacer davantage les obstacles liés à l'espace et au temps". C'est au sein de ce conflit entre l'oralisme et la langue des signes que plusieurs acteurs sociaux sourds vont choisir leur forme préférée de communiquer avec le monde et les autres. Ainsi, le conflit est aussi lié au droit d'un sourd d'être un acteur social qui assume son identité sourde, avec la possibilité de faire des choix, dans ce cas porter ou non un implant cochléaire. D'après Simmel (1999, p. 265) "si toute action réciproque entre les hommes est une socialisation, alors le conflit, qui est l'une des plus actives, qu'il est logiquement impossible de réduire à un seul élément, doit absolument être considéré comme une socialisation". Sur la thématique du conflit chez Simmel, Watier (2003, p. 72) approfondit la discussion affirmant que "la socialisation par la lutte crée une unité qui transforme les relations de cette unité à la société globale; sans le conflit sur la définition de l'existence sociale, le groupe n'aurait eu qu'une existence stigmatisée".

Nous comprenons que les luttes menées par les sourds avec le but de s'intégrer dans la société, par exemple dans le marché du travail, leur permettent de prendre conscience de leurs capacités. Le conflit n'est pas une éventualité dans la vie des sociétés, il en fait partie intégrante.

Il nous faut dire que le conflit entre l'utilisation de la langue des signes et de la méthode orale remonte à loin. Selon Mottez (2006, p. 45): 
Pendant le XIX ème siècle, deux méthodes se sont néanmoins affrontées pour l'éducation des sourds. Si certains suivaient celle par laquelle l'abbé de l'Epée avait frayé le chemin à l'éducation de masse des sourds, d'autres, suivant S. Heinicke, de Leipzig, préconisaient un enseignement exclusivement oral. Estimant qu'on ne pense que pour autant que I'on parle, ce dernier soumettait tout enseignement à l'apprentissage préalable de la parole - exercices d'articulation et de la lecture labiale.

De nos jours, une discussion sur l'implant cochléaire révèle bien la dissension entre les professionnels qui parlent de l'importance de l'oralisme pour les sourds et ceux qui soutiennent la langue des signes comme la première langue des sourds. D'après nous, l'implant cochléaire montre aussi l'avancée des technologies et de la médecine relatifs aux possibilités de transformations corporelles selon la norme établie, dans ce cas le modèle de la majorité entendante. Ainsi, I'implant cochléaire est l'instrument, fruit des avancées technologiques, qui va placer l'être sourd à la frontière entre la surdité et les entendants.

Au cours des dernières années, de grands progrès ont été réalisés en matière de prothèses auditives, de même qu'on a assisté à de remarquables avancées dans les chirurgies spécialisées et méthodes de rééducation de toutes sortes, dans le but de développer l'audition des personnes sourdes, autrement dit de réduire la déficience. Tous ces progrès encouragent la société actuelle, tel un phénomène de mode, à privilégier les nouvelles technologies d'information et de communication pour se limiter à «traiter » la surdité sur un plan médical.

En vérité, les expressions corporelles développées par la population sourde ne sont pas considérées par la société des entendants. Selon Le Breton (2011, p.
158) "le corps est comme la dimension impudique de la parole, celle qui s'impose au regard, et dont il convient d'atténuer la présence en la subsumant sous les codes de discrétion en vigueur. La langue des signes paraît à l'inverse un hymne au corps et au visage, elle suscite la gêne des entendants pour qui seule la voix est digne de soutenir le langage".

Dans la société occidentale, le corps est nié dans ses expressions de langage et remplacé immédiatement par la parole, par la verbalisation orale et écrite comme une forme primordiale d'expression humaine. Par contre, chez les sourds, le corps est une forme d'expression première avec une grande importance des expressions faciales dans le langage gestuel; donc les codes corporels, gestuels, constituent une langue précise, la langue des signes.

De nos jours, des acteurs sociaux touchés par la surdité pouvent, au cours de leur existence ou dès la naissance, comprendre les sons extérieurs, en raison du développement des technologies d'information et de communication. L'appareil qui va permettre la possibilité de cette connaissance peut être un implant introduit dans le corps de l'acteur social sourd et à travers lui, les sons du monde extérieur peuvent être codifiés. Ainsi, une nouvelle forme d'appréhension du monde est possible chez les sourds. Du point de vue médical, le transplant cochléaire est utilisé avec succès. Néanmoins, la question sur laquelle nous mettons l'accent est de comprendre les conséquences possibles de cet acte sur un acteur social? Quelles frontières sont de fait redessinées ou déplacées avec l'utilisation d'un implant cochléaire? Alors, nous ouvrirons ce dialogue avec les cas qui suivent.

\section{LES CAS}

La présente étude aborde le cas de deux jeunes sourds qui ont mis un implant cochléaire. Le premier cas, est un garçon 
oralisé de treize ans qui continue d'utiliser l'implant cochléaire de même qu'il utilise la langue des signes pour interagir avec le monde et les autres. Par contre, le deuxième cas, est celui d'une jeune fille non oralisée de onze ans qui a décidé de retirer l'implant cochléaire et a choisi d'utiliser seulement la langue des signes pour communiquer dans son quotidien. Il est vrai que l'histoire des sourds, comme celle des autres groupes sociaux, a été construite à partir d'une série de faits violents qui ont eu des conséquences sur leur langue. Comme le dit Stiker (2005, p. 205) "la difformité des corps, les troubles de l'esprit, les privations de sens, ont toujours inquiété les groupes sociaux, tout comme le sexe, le pouvoir, l'échange, la mort ou les ancêtres".

Nous soulignons que les informations et les dialogues des deux acteurs sociaux sourds ont été viabilisés par une interprète de langue des signes française LSF. Les noms des participants de cette recherche ont été changés pour protéger leur identité. Ainsi, les identifications utilisées dans cette étude sont des noms d'emprunts.

La langue des signes n'est pas universelle. Chaque pays a sa langue des signes. De plus, la langue des signes, dans les différentes régions d'un pays, peut présenter une variation, c'est-à-dire que nous pouvons constater une variation sociolinguistique dans la langue des signes. Par exemple, nous pouvons percevoir une variation dans une expression de la langue des signes si nous faisons une analyse entre la région de l'Alsace et la région du Poitou-Charentes. Cela nous montre la richesse de cette langue qui n'est pas encore reconnue par une partie de la société actuelle.

A propos des données présentées, nous pouvons citer deux terrains sur lesquels s'articule la présente étude: le sourd qui a choisi la frontière entre l'oralisme et la langue des signes, avec des conflits spécifiques, et le sourd qui a choisi la langue des signes comme langue principale.

Nous appellerons l'acteur social du premier cas Michel, qui est un garçon sourd de naissance. Ce garçon a mis l'implant cochléaire à l'âge de 4 ans. A ce moment-là, le jeune Michel vit avec les sons transmis et captés corporellement par l'appareil implanté. D'après l'interprète en langues des signes, quand il a été questionné sur ce quotidien avant et après l'implant cochléaire, Michel a dit:

\begin{abstract}
Avant de faire l'implant cochléaire, j'avait les petits appareils, mais je n'entendais rien du tout. L'oreille droite ça allait à peu près, par contre l'oreille gauche je n'y entendais rien du tout. Aujourd'hui, j'oralise avec les entendants, et s'il y a des sourds qui en ont besoin, je vais utiliser la langue des signes.
\end{abstract}

Nous avons observé que pour les sourds oralisés qui maîtrisent la langue des signes, lorsqu'ils sont en interaction avec d'autres sourds, c'est la langue des signes qui conduit la communication entre eux. Comme le dit Le Breton (2011, p. 154) "la parole n'est pas la seule modalité du langage. En fait l'enfant sourd participe d'une communication globale, vocale avec ses proches entendants, et signée avec ceux qui partagent leur surdité".

Michel avait obtenu de bons résultats, environ neuf ans après la pose de l'implant. L'implant cochléaire l'aide à oraliser. Interrogé sur sa préférence entre la langue des signes et I'oralisation, Michel n'a pas su répondre. Néanmoins, il nous fait comprendre que cela ne le dérange pas quand il a besoin d'oraliser. Sur ce point, écoutons la réponse de Michel:

Entre la langue des signes et la langue orale, il n'y a pas un mode de communication que je préfère plus. J'utilise les deux. Chez moi, 
j'utilise la langue orale. Mes parents ne connaissent pas la langue des signes.

Nous mettons l'accent sur le fait que pendant l'entretien, Michel a préféré utiliser la langue des signes et a demandé la présence d'un interprète en langue des signes. D'après nous, la langue des signes est la principale forme d'expression des sourds ainsi que leur identité dans le monde. Interrogé sur la forme de communication dans son quotidien, par exemple dans l'école et dans d'autres lieux sociaux, Michel a souligné qu'il ne réussit à interagir qu'avec l'oralisme. Il nous a dit que:

Parfois, il y a des gens qui ne me comprennent pas. Je ne sais pas pourquoi.

C'est vrai que les avancées dans les nouvelles technologies d'information et de communication, dans ce cas l'implant cochléaire, peuvent rendre possible pour les sourds la compréhension des sons. Ainsi cet acteur social va entrer dans une partie significative de l'univers des entendants, l'univers de la sonorisation, qui le conduira à l'oralisme. De cette façon, à partir du moment où le sourd établit une communication orale avec l'acteur social entendant, c'est ici que se marque la frontière pour cet acteur social sourd, entre le sentiment d'être sourd et celui d'être entendant. Dans ce cas, la communication est l'objet d'intérêt et l'implant cochléaire et l'oralisme délimitent la possibilité d'établir la communication, construisant ainsi cette frontière individuelle. Simmel (1999, p. 609) remarque que:

Dans la majorité de tous les rapports entre individus comme entre groupes, la notion de frontière revêt sans doute de l'importance d'une façon ou d'une autre. Partout où les intérêts de deux éléments se portent sur le même objet, la possibilité de leur coexistence dépend de la délimitation de leurs domaines respectifs par une frontière au sein de cet objet - que celle-ci soit une frontière juridique qui marque la fin du conflit, ou une frontière de puissance qui en indique peut-être le début.

Dans ce passage, le sourd continue à entendre les sons et à transmettre sa voix qui va avoir besoin d'être toujours améliorée dans les cabinets des orthophonistes. Michel vit cette frontière qui ne paraît pas le déranger. Par contre, lorsqu'il rencontre des amis sourds, il va s'approprier de la langue des signes en vivant son identité sourde.

Bien différent de Michel, Lyse, le second acteur social sourd présent dans cette étude, après avoir éprouvé l'expérience de la frontière entre l'être sourd et l'être entendant, a choisi de retirer l'implant cochléaire. Elle a adopté la langue des signes comme sa première langue. L'implant cochléaire lui a été mis à l'âge de un an et demi. Elle a vécu jusque vers neuf ans et demi avec l'implant cochléaire.

En questionnant Lyse sur la raison de son choix de retirer l'implant cochléaire, elle nous a dit que:

Ça (l'implant cochléaire) me faisait des bruits, j'avais mal à la tête. J'entendais un tout petit peu les sons, les paroles de gens, mais en fait la communication ne passait pas. Avec mes parents, en fait, je leur montre des livres de langue des signes, pour qu'ils apprennent les signes parce que mes parents sont entendants. Voir les signes pour que la communication soit plus facile. Donc je leur apprends, au fur et à mesure ils apprennent les signes. 


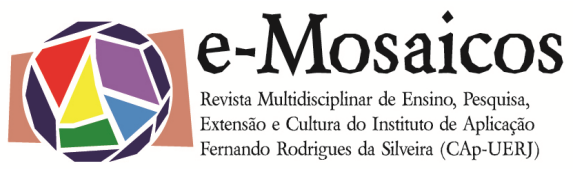

Pour Lyse, le monde est trop bruyant et les sons ont perturbé la construction de ses pensées. Lyse a fait valoir qu'elle se sent tellement mieux sans le bruit du monde extérieur. Après être passée par l'expérience d'écouter, d'avoir un contact avec le monde en utilisant également le sens de l'ouïe, Liz a choisi de continuer et d'améliorer son expression corporelle par les signes corporels. Les sons et l'audition n'appartiennent pas à son monde. Ainsi, elle a choisi le silence, le monde visuel et surtout la langue des signes pour interagir avec le monde et les autres.

Pour se rendre compte clairement de ce que nous voulons dire sur les frontières de la communication chez les sourds, il faut établir les relations entre I'oralisme et la langue des signes assujettie aux avancés technologiques et à la politique actuelle d'inclusion des sourds dans la société. Ce qui ressort surtout des témoignages de Lyse et Michel, est la possibilité de faire des choix. Les sourds doivent avoir le droit de choisir leur forme de communiquer avec le monde et les acteurs sociaux entendants. Ainsi, la société actuelle doit rechercher les voies qui facilitent son adaptation au monde des sourds. La société aussi doit s'adapter à la communauté sourde, par exemple en maîtrisant la langue des signes.

Cette discussion scientifique est aussi politique et elle établit des frontières avec d'autres actions, comme la connaissance historique et les normalisations sociales, entre la reconnaissance des différences et la relation avec la diversité des formes de communication dans le monde. Dans cette perspective, la dimension symbolique et réelle de la communication, les frontières entre le monde des sons des entendants et le silence communicatif des sourds sont des sujets qui nécessiteraient d'être débattus dans l'actualité.

D'après nous, chaque acteur social a des sentiments, des émotions qui fondent son identité et déterminent son groupe social. La communication de la com- munauté sourde passe par la perception des événements, les expressions corporelles et la langue des signes.

\section{EN GUISE DE CONCLUSION}

La société actuelle nomme encore les personnes sourdes "sourds-muets" ou "muets". Etre sourd n'est pas être muet. Il faut réparer cette erreur, qui participe à l'exclusion des sourds, considérés comme inaptes à comprendre, à communiquer, à penser, et à interagir avec le monde et les autres.

En réalité, il faut abandonner toute vision réductrice concernant l'être humain et tourner nos regards vers la subjectivité et la sensibilité humaine. En effet, la société actuelle vit des moments de changements intenses et successifs. À tout instant, dans ce monde en constante mutation, de nouvelles propositions, de nouveaux pouvoirs, de nouveaux regards, de nouvelles cultures, de nouveaux groupes sociaux, de nouvelles identités surgissent ou sont repensés, recréés, reformulés.

Le quotidien des sourds qui utilisent la langue des signes et celui des sourds oralisés n'est pas du tout de même nature. Les deux enfants sourds qui ont participé à la recherche communiquent avec le monde et les autres de manière différente. Ces enfants sont dans une nouvelle frontière. L'implant cochléaire tente de rendre possible une nouvelle existence corporelle en associant diverses technologies.

D'après nous, c'est la langue des signes qui donnera aux sourds leur identité, en dépit du fait que de nombreux éducateurs soutiennent la méthode oraliste pour les sourds et ne considèrent pas la langue des signes comme la première langue pour les sourds. Les avancées technologiques ont facilité l'inclusion sociale des sourds avec toutes les ressources du monde de l'informatique. Par contre, la 
société exige que les sourds s'adaptent à l'idéal des entendants.

Les avancées des nouvelles technologies de l'information et de la communication, par exemple l'implant cochléaire, amènent les sourds dans une zone frontière. Les sourds qui ont l'implant cochléaire ou qui sont appareillés, tendent à percevoir les sons car le but principal de ces technologies est de favoriser I'oralisation. Cependant, il faut dire que cette compréhension des sons ne donne pas aux sourds la condition d'entendants. En vérité, c'est un sourd qui utilise des appareils pour comprendre la société entendante.

Les auteurs du présent article assument une position critique face à l'oralisme comme unique forme de communication pour les sourds. Imposer I'oralisme aux sourds et croire qu'il est la principale voie pour les sourds pour interagir dans une société entendante, est, selon notre point de vue, une forme actuelle d'oppression des minorités.

\section{RÉFÉRENCES BIBLIOGRAPHIQUES:}

AUGÉ, Marc. Pour une anthropologie de la mobilité. Paris: Éditions Payot \& Rivages, 2009.

LE BRETON, David. Eclats de voix: une anthropologie des voix. Paris: Métailié, 2011.

JUTEAU, Danielle. L'ethnicité et ses frontières. Montréal: les Presses de I'Université de Montréal, 1999.

MOTTEZ, Bernard. Les sourds existent-ils? Paris: L'Harmattan, 2006.

SIMMEL, Georg. Sociologie: études sur les formes de la socialisation. Paris: Puf, 1999.
STIKER, Henri-Jacques. Corps infirmes et sociétés. 3e édition. Paris: Dunod, 2005.

ZRIBI, Gérard; POUPEE-FONTAINE, Dominique. Dictionnaire $d u$ handicap. $5 e$ édition. Rennes: ENSP, 2004.

WATIER, Patrick. Une introduction à la sociologie compréhensive. Belfort: Circé, 2002.

- George Simmel sociologue,

Belval, Circé, 2003.
Recebido em: 15/03/2017

Aceito em: $10 / 04 / 2017$ 